Journal of Teacher Education for Sustainability, vol. 23, no. 1, pp. 150-165, 2021

\title{
More Personal Knowledge for More Sustainable Higher Education
}

\author{
Ilga Salīte, Ilona Fjodorova and Inese Butlere \\ Daugavpils Universiy, Daugavpils, Latvia \\ Oksana Ivanova \\ Riga Technical University, Riga, Latvia
}

\begin{abstract}
The article aims at considering the characteristics of the Anthropocene era from a broader perspective and emhasizing the possibilities of pedagogy to reduce the current unsustainability of higher education. The relationships between human and nature are affected by the wicked problems. In particular, they are influenced by intermingling of anthropocentrism, egocentrism and egoism, which affect these relationships by causing changes in the human being, i.e., changes in sensitivity and sensibility occur that are naturally necessary for human adaptation to the environment.

For the case study, we have used the processes taking place at the phenomenon level, which are known as the first-person perspective (1PP) characterized by an active and dynamic connection with the environment. It should be noted that 1PP cannot be observed from a distance, as there are a number of interrelated processes that begin with the effects of the environment, the formation of the "self" and the formation of the self-image. It follows that complex consciousness in the 1PP is implemented within an egocentric reference system, where experience is structured according to the image of the human body. The article will present the findings of 1PP study obtained by performing two tasks: (1) development of a personally significant view of a 1PP researcher on the phenomenon of sustainability from an egocentric perspective and (2) involvement of the 1PP researcher in adaptive learning suitable for pandemic conditions. We would like to stress that the case study has been developed as our reaction to the intensification of unsustainable development in the relationship between nature and human, which has been initiated and maintained by the tangle of Anthropocene problems. From the pedagogical perspective, we looked for an opportunity to use the basis of the natural origin of human and nature relations to maintain more integrated activities and more personal knowledge, so that the development of more sustainable higher education could be promoted by means of personal experience. Through the pedagogical lenses of phenomenology, we identified the need to reduce the impact of the Anthropocene tangle through the use of more natural methods. We also recognized the need to use the 1PP and adaptive learning opportunities more carefully. In this way, pedagogy can gain new experiences for a more holistic understanding of individual experience and enhance the
\end{abstract}


use of the unique abilities of adaptive learning to prototype more personal knowledge in order to build more sustainable higher education.

Key words: Anthropocene era, human and nature relations, limiting effects of unsustainable education, egocentric reference system, first-person perspective, prototyping of adaptive learning, personal knowledge

\section{Introduction}

The aim of the article is to look at the characteristics of the Anthropocene era from a broader perspective and to highlight the possibilities of pedagogy to reduce the current unsustainability of higher education. At present, the theme of Anthropocene and higher education has become topical. The idea of Anthropocene has been used in the study as a context in its broadest sense. Anthropocene has acquired the status of an era in the $21^{\text {st }}$ century and has already been given several designations: an era of uncertainty, wicked problems, unsustainable development. All of these names are associated with the quality of unsustainability that has manifested itself in our planetary system. We have expressed our concern about this issue in the Journal of Teacher Education for Sustainability (Salite et al., 2020). Currently, there is confusion surrounding the relationship of human beings and nature, in which the manifestations of anthropocentrism, egocentrism and egoism are intertwined, creating unpredictable feelings of insecurity through and under the influence of information space because there has been a change in the relationship between sensitivity and sensibility, which in a natural way ensures buman adaptation to the environment. From the perspective of ecology and sustainability, this problem was studied in teacher education, which was evaluated within the framework of the Doctoral Thesis in 1993 (Salite, 1993). Since then, the problem of unsustainability of anthropocentrism and the Anthropocene era has become a global issue and in recent years the theme of an ecological approach has returned to it, which is evaluated from the perspective of relation between higher education and Anthropocene (Stratford, 2019).

The Third UNESCO World Conference in Berlin is one of the largest events in which higher education institutions (HEIs) have been encouraged to address this issue, despite the fact that the HEI involvement in the implementation of sustainable higher education has a long history. Not everything that has worked in previous decades is yielding results now. The changes at the beginning of the $21^{\text {st }}$ century have not brought clarity to solving problems. It is possible that now all those who see the threat of unsustainable development and education affected by the pandemic have come to the conclusion that human health and planetary health are interrelated and that complex man-made problems can be addressed through the human-nature relationship. This holistic view has recently been echoed in all global and local events and activities. It was highlighted at the UNESCO conference in Berlin and would be emphasized in all events taking place after the conference. Pope Francis also calls for a radical transformation of international relations, since it emphasizes the deep implications of environmental issues and call for "Integral Ecology" in environmental relations (Ferrare, 2019).

Integral thinking and integral learning are ancient themes in philosophy, antropology, education and holistic pedagogy (Brigman \& Lewis, 2017; McKenna, 2012). The implementation of the theme has been "stuck" in various ways, especially in the $21^{\text {st }}$ century, and it is usually in the interests of somebody and it is usually a minority interest. 
Unsustainable democracy (Salìte et al., 2020, p. 4) has long offered humanity situations that run counter to Aristotle's view that decision-making should be based on what will be a good solution for the majority (Aristoteli, 1985). Examining this view on decisionmaking from an Anthropocene perspective, we have realized that the issue of "seeking benefit for the majority" is not an essential feature of the Anthropocene age (Salite et al., 2009). The decision can be made by those who are in a minority grouped around an issue and are able to organize the adoption of a conscious or unpromised issue that will give some benefit to the minority. Mankind has often fallen into this type of trap when it comes to agreeing on an idea that is important to humanity or nature, but the explanation or visual representation of the idea, or the metaphors used to explain the idea and, of course, the approaches and methods of implementing the idea create a different, even contrary, effect.

In recent years, sustainable development and its inherent three dimensions are not visualized with the Venn diagram in texts, as the offer has disappointed those who perceive the world as an open, complex and dynamically adaptive and evolving process, in which integral ecological thinking and action processes are viewed from the natural origin perspective, where ecological / environmental relations include culture and social relations and the inseparability of human activity and consciousness. In the visual representation of the Venn diagram, disregard for scale and relationship and too free representation of the sustainability phenomenon, ignoring people's ontological experience and sense of reality, have not gone unnoticed: most people in the $21^{\text {st }}$ century global society have not understood the idea of sustainable development and the sustainability phenomen even until the end of the first Decade of Education for Sustainable Development (2015) and the situation is still ongoing. It seems that in the last two decades of the $20^{\text {th }}$ century and at the very beginning of the $21^{\text {st }}$ century, this issue was understood by the majority. At that time, a more holistic perspective of pedagogy was sought. It was sought in both reality and ideas, and it awakened the opening of spiritual creative forces in society and education. This could not be done without the search for integral thinking, in which the world and human being are an indivisible whole, embedded in a world open to development. Through a more holistic perspective that developed in ecological / environmental education and pedagogy, the relationship between human being and nature was more natural and understandable at the time (Pigliucci \& Kaplan, 2006).

From participation in action research at the beginning of the $21^{\text {st }}$ century, we have seen a decline in the importance of the holistic approach in education and science in general. The focus on the use of an integrated approach in pedagogy and the pedagogy of holism itself at the beginning of the $21^{\text {st }}$ century were overshadowed by the substantiation of educational science, which was sought by conceptualizing educational practice. The basis for a competency approach has been recently sought. In the search for pedagogical science in the context of projects and social sciences, the role of pedagogy as a science has been diminished.

\section{The Tangle of Wicked Problems in the Antropocene}

Let us look back at the Anthropocene perspective offered in the introduction to the article. We have focused on a complex, wicked problem "tangle", which we have highlighted as important in the context of the current human-nature relationship. This "tangle", in our opinion, is the result of Anthropocene, which also acts as a limiting 
force for sustainable development with a complex root based on unsustainability. The question of this "tangle of Anthropocene problems" must therefore be seen in the light of the reasons for its unsustainability.

One issue with "the tangle of these problems" is the impact on people. The effects of Anthropocene are now causing humans to experience a great deal of doubt and confusion over issues that have not seem so dangerous in previous years. It seems that confusion arises from the intermingling of different issues, which create the tange of unsolvable problems. In this article, we consider a tangle limiting sustainable development (the overlap and confusion of anthropocentrism, egocentrism and egoism) at the very core of Anthropocene. It destroys the fundamental basis of human adaptation with changes in human sensitivity and sensibility. Observing what is happening in social communication, one gets the impression that people's perception and sense of life are hopelessly confused and it is not possible to understand and recognize the issues of reality. In times of crisis, it is probably natural that there is a lack of experience in understanding issues and the meaning generated from experience, which helps find a way out of problems and continue on the path that will give hope and faith to the future of humanity.

The other easily identifiable issue of the Anthropocene problem tangle is the impact on the unsustainability of education. Through the acquisition of higher education and the fundamentals of educational research and alienation from the implementation of specific tasks of pedagogy, education is directed towards non-ecological and unnatural learning, by diminishing the role of evolutionary experience and biological origin of many phenomena (Morton \& Mylopoulos, 2020; Metzinger, 1995).

Currently, the use of the piecemeal approach from the research perspective stands out in educational research, which does not always take into account the formation of living experience units in complex life processes. The intentions of research become especially unclear, in which pedagogy is not used as a science and the authors of the research hide behind specific problems of educational science or other non-pedagogical interests of the researchers themselves. Then, behind the interests of educational science and funded educational projects, the pedagogical incompetence of researchers can be hidden through a piecemeal approach. This refers to researchers who have never been interested in the specific tasks of pedagogy, even denied pedagogy as a science. But there is also an intentional or unintentional deception hidden in "competition" that is maintained in piecemeal research. It could also be called as insufficient retaining and long-term preservation of the understanding of the speculative function of pedagogy in educational research during the Anthropocene age. Its basis can be found in the explanation of the speculative function of pedagogy, i.e., pedagogy is related to all the sciences, fields of activity and various ideas generated by humanity and they can be used for the achievement of the specific mission of pedagogy. This explanation can be based on traditional linear understanding and also on nonlinear studies of complex problems. Their implementation can be recognized by the choice of integration approach or research approach used in the teaching / learning process or research activities. These are more often the interdisciplinary integration approach and the transdisciplinary integration approach, which are also used to explain the speculative nature of pedagogy, respectively. Looking for a deeper insight, it is easy to discover that the interdisciplinary approach will be based more on the complicated approach in both the educational process and educational research, while the transdisciplinary approach will use the complex approach. Today, 
any good educator and modern researcher in educational research, and especially for ESD, will understand that pedagogical science is by nature holistic, transdisciplinary, and proactive. Through participation and learning, pedagogy evaluates individual and group experiences and the meanings they create, which are revealed in the choice of types of survival, sensitivity-sensibility changes and living activities experienced in nature and in recognition of one's way of life through continuous prototyping of one's personal identity (Hampton, 1991).

The current problem of the Anthropocene era is related to the lack of a basis in the philosophy of science, in which interdisciplinarity and transdisciplinarity are identified by their deeper meaning. This is currently the case in pedagogy, where the recognition of complicated and complex approaches in theory shows that these approaches must be seen from the perspective of pedagogy as a complex science. However, scientific articles are frequently based on the complicated approach, which in recent years has been hidden only by the attraction of keywords characteristic of the complex approach. Participation in conferences in different European regions, especially in calls for research projects for the coming periods, provides an opportunity to be confronted with the different policy perspectives offered in different European regions. In some conferences, the methodological foundation of research resorts to interdisciplinarity, in other project applications there is a tendency for transdisciplinarity. The aim of these conferences was only one: to promote the drawing up of applications for modern scientific research projects. The composition of the member states was different and, in fact, these were two different orientations that were proposed as two different ways to use integration. One of them will clearly focus on the use of the piecemeal approach from the perspective of a complicated approach and will serve the requirements of the Anthropocene age. The second can serve the interests of proactive pedagogy, provided that the project promoters and experts themselves have delved into the deeper essence of the transdisciplinary approach. In highly cited publications and informal conversations with participants of these two orientation conferences, it is easy to come across the acknowledgment that the participants themselves do not see and have never seen the difference between interdisciplinary and transdisciplinary perspectives. In our opinion, this is an important feature of Anthropocene that these cases of integration are not yet recognized by some researchers, as education and social sciences are still dominated by a complicated approach (during the holism period, in the 1980s and 1990s, this approach was often referred to as the puzzle approach); moreover, at present the complicated approach is reinforced by the use of excessive statistical techniques (Honkela et al., 2005). Every year, the names of the test authors, the data processing programs used are increasingly highlighted, and the conclusions about the significant qualitative benefits of the study become shorter and shorter. The use of statistical and systematic methods is increasing, but there is still a need for more complicated, synthesizing and adaptive research to find a more sustainable path, as there are growing concerns about the Anthropocene wicked problems, which have taken on catastrophic shapes.

Students of Master study programs at higher education institutions have two opportunities in such circumstances: (1) to learn to recognize different approaches using available sources and to develop their personal view of educational research for ESD in order to participate in profound research of the issue under consideration or (2) to integrate in sometimes "muddy waters" of Anthropocene research and seek survival opportunities there. 
Survival in the Anthropocene age is difficult not only for students, but also for outstanding authors who started to form a theoretical basis for both sustainability phenomenon and transdisciplinary and/or interdisciplinary approaches, but the initial circumstances did not provide a sufficiently recognizable basis for approaches. As new approaches appear, it is natural that the features can be identified by talking about the most characteristic features of the interdisciplinary approach and unexpectedly the features of the transdisciplinary approach appear below the explanations of the former approach. Recognizing complex phenomena is patient work of expanding one's personal experience, generating personally significant meanings, and constructing personally significant knowledge. The situation is currently affected by a tangle of problems localized in the Anthropocene nucleus.

The third easily identifiable problem of the Anthropocene tangle is its easy transfer through the communication and technology environment and its use. It is a catching phenomenon and it often causes internal poisoning or develops as self-poisoning that comes from the communication environment.

The mentioned cases point to the Anthropocene environment, which is based on the constraints of a tangle of wicked problems, which we can recognize as unnatural, because the given cases are narrower than the perspectives already known to humanity, which go beyond anthropocentrism. In the three cases of the Anthropocene solutions mentioned above, humanity has long recognized ecocentrism and deep ecology, the renewal of culture through the experience of individuals and the formation of meaning and self-development generated on its basis, which is a condition for the search for the highest goal of the human species. Humanity has sought to know and explain the world more widely, but in the Anthropocene environment these efforts are undermined by the influence of its limiting forces.

\section{Case Study: Choice from the Pedagogical Perspective}

The pedagogical view of the goal of education as the attainment of humanity is based on the fact that humanity is a natural phenomenon, i.e., this phenomenon has a biological and evolutionary origin (Rosenberg, 2004; Perry et al., 2010; Graziano, 2019; Snider, 2017; Thompson, 2003). At present, the formation of this phenomenon is interpreted as a complementarity between ecological and evolutionary processes (Stratford, 2016, June 11). However, the biological basis of the human phenomenon cannot be accepted by all, as it requires a broader and deeper understanding of natural phenomena. This cannot be explained by a social approach alone. This requires an integral ecological basis for natural phenomena.

The new virtues of the Anthropocene that are brought into education are based on increasing the pressure of the social and economic environment. This can be disproportionately strong and lead to a reduction in environmental commitments and ownership. In a critical period when natural adaptive learning and path choices are being pushed out, it is important to maintain and develop pedagogical interest in the use of natural phenomena in education (Wong, 2012). This is not an easy task in the current circumstances, for example, the cohort approach emerged as a result of the new methods and approaches proposed for the implementation of the New Competency-Based Approach to Curriculum Reform. Educators with professional competence were shocked by such an approach in pedagogy, but technically oriented educators and supporters of faster 
technology implementation did not understand the essence of this situation. It seems that in the coming years there will be many more offers to supplement the arsenal of educational methods and approaches, which will not come from the perspective of pedagogical science. It is important for the development of pedagogy that these innovations do not erase the natural ability necessary for the development of humanity to perceive natural phenomena in the real world through the sensitivity and sensibility of the environment and to find the foundations of one's identity.

The issue of recognizing natural phenomena raises some concerns in pedagogy. In our opinion, this will also contribute to the understanding of the "self" phenomenon. Even now, if we look at Anthropocene interests from a greater generalization, we can see that the tangle of Anthropocene wicked problems is focused on the "self" phenomenon and its deformation in terms of social survival and the illusion of unsustainable human success in space mission. But should not we look here at the readiness to react authentically through adaptive learning from personal experience and the choice of reasoning for decisions in favor of a goal important to humanity and participation of human beings in the implementation of this goal, accepting it as personally important?

Personally important things are related to pedagogical interest from the perspective of phenomenology. Through this perspective, phenomenological research develops definitions derived through the interpretation of living experience. Phenomenology does not exclude researchers' personal experience in interpreting the phenomenon. The structure of living experience then becomes the basis of the "self" experience, and in it the living experience and the meaning generated from it refer to the "self" and are reflected in the individual's identity and the way the individual handles everything encountered in the actions and interactions.

At the phenomenon level, the above-mentioned processes, from the pedagogical perspective, do not require the usual measurement or comparison. In these cases, adaptive approach methods will be needed, as statistical measurements will not provide an answer to what is happening. Pedagogy in these cases will seek help for pedagogical situations that are happening, will allow them to happen and will try to understand what is happening. At the phenomenon level, processes take place that are accessible to individuals and have an internal natural structure. These processes cannot be seen from the sidelines. There is a need for pedagogical techniques that allow understanding what is going on. Pedagogy usually uses indwelling, identification, or empathy in such cases. These techniques involve entering the unconscious and through the state of indwelling experience and meaning come out of the silent unconscious zone as personal knowledge (Polanyi \& Prosch, 1975). Dwelling on the unconscious and indwelling give personal knowledge that deepens our knowledge of living beings that become personal (Polanyi \& Prosch, 1975; Wright \& McCarthy, 2010). In the context of pedagogy, this is a topic that leads to the use of natural processes in integrated education. There were periods when the idea of indwelling was used to compare the idea of sustainability with the ability given by nature, in which one could easily recognise the healthy body systems. Recognition of a healthy plant or animal or action is given by nature and strengthened in the process of evolution. The ideas of healthiness and sustainable development were successfully used in the Gothenburg Global Colloquium (2004). 


\section{Case Study Design Choice}

The case study has been developed as our reaction to the intensification of unsustainable development in the relationship between nature and human, which is initiated and maintained by the already mentioned tangle of Anthropocene problems. We have formulated our proposal as a perspective using the inseparable connection of human and environment origin, which is the basis for the existence and development of integral action and thinking. This is a certain truth that highlights the fact that the action of natural phenomena begins with the interaction of the individual and the complex environment and the individuals ability to respond to information received from the environment (Revonsuo, 2006). In pedagogy, it is also seen as a unique individual's ability to adapt in order to survive in a complex environment.

Creating the case study design, we have used the processes taking place at the phenomenon level, which are known as the first-person perspective (1PP), which is characterised by an active connection with the environment. It gives a personal direction and meaning to human life (Zanker, 2010; Zappala, 2009) that maintains a philosophical view, in which consciousness and experience are inseparable, and consciousness is complex. According to Revonsuo (2009), complex consciousness in the first-person perspective (1PP) is implemented within the framework of an egocentric reference system, where experience is structured according to the image of the human body itself (Revonsuo, 2009). Within an egocentric frame of reference, location and distance among perceptual units are spatially represented in a person's personal relationships and in their body image, so in our daily thinking we localize our "self" within our body.

The first-person perspective cannot be observed from the sidelines. In this perspective, there are a number of interrelated processes that begin with the effects of the environment, the formation of the "self" and the formation of the self-image, which Revonsuo (2009) metaphorically calls selfie formation, which by its nature acts as a unique node of adaptation. There the sequential separation of the individual processes taking place is not important, but it is important that they take place and in an inaccessible way to observation, an identity emerges from the formation of experience and meanings, which gives an individual's activity a more personal sense of identity and more personal knowledge.

The study is based on the 1PP with its characteristic egocentric reference system. The article will present the results of 1PP study, which were obtained by performing two tasks:

(1) development of a personally significant view of a $1 \mathrm{PP}$ researcher on the phenomenon of sustainability from an egocentric perspective, and

(2) involvement of the 1PP researcher in adaptive learning suitable for pandemic conditions.

To fulfil the first task, we proposed a methodology, the use of which was appropriate after the distance learning approach came into force. It is one of the simplest research methods "Pairwise Comparison". It can be easily adapted to the needs of the action research, as it opens up opportunities to clarify the participant's personal experience and personal meanings on one of the issues that is topical in the action research. Within the framework of the research question, the participants usually identify and evaluate the personally significant values or features of a phenomenon. In the evaluation process, the participant evaluates the identified features against each other by comparing and 
evaluating each identified feature with all the others. In the evaluation process, the point is given to the feature that is "stronger" or more pronounced in a particular case.

The first task of the 1PP study involved the question: "How do I understand the idea of sustainability in the context of my life or education for sustainable development?"

Each feature was given a score in the evaluation process, which became the subjective value of the feature. From the first-person perspective, the researcher selected 4-5 features and awarded them the highest score, and, if necessary, the features could be changed to the ones more important to the researcher. In essence, it was a selfie obtained in the 1PP egocentric reference system.

To fulfil the second task, we offered to use doctoral theses in teacher education elaborated from 2006 to 2015 at Daugavpils University, with a view to promote the reorientation of teacher education towards environmental and sustainable education. We chose research conducted on the methodological basis of holismpedagogy, which could help in the acquisition of university experience that is based on tradition in the context of the institution. Within the framework of the study, we offered four summaries of doctoral theses, which the research participant used as teaching aids and through which broadened her personal view on the phenomena of ecology and sustainability.

The researcher evaluated summaries of the doctoral theses by identifying the proposed sustainability ideas and integrating or synthesising them in a personally relevant view of the sustainability phenomenon. At the end of the task, the ten most important features for the researcher had to be selected and evaluated. The evaluation of the features was performed in the same way as in the first task.

\section{Research Findings and Discussion}

During the first stage of the study, we have taken into account Revonsuo's (2009) finding that this type of study is not available to observers, and pedagogy also knows that the results of this study will be available by observing the results after the study when they become recognizable in action and in verbal expressions, in which changes can be identified. However, information about 1PP experience can be used if the researcher shares this information for use in a 3PP study. For this purpose, we have offered the researcher to prepare visualized information about the performed tasks after the completion of the tasks of the first stage. In the visual structure, we have identified a similarity with the framework of the egocentric reference system and the possibility to place the features with the highest score in the center sectors and the features with low score in the periphery of the chart (white squares). In the real process, depending on the actualized experience or meanings, the place of different perceptual structures may change. They can change place and take up different space. In the study, this natural living scene has been offered as selfie only in the form of snapshots.

Meaning is subjective in nature because the individual determines it by accepting in $1 \mathrm{PP}$ what is meaningful. It creates a sense of the world that becomes recognizable in behavior (Polanyi \& Prosch, 1975).

The visualized selfie moments offered by the researcher were 1PP subjective insights. According to Revonsuo (2009), one person cannot be involved in 1PP and 3PP at the same time. Therefore, during the second stage of the study, a third-person perspective (3PP) was used, based on the 1PP study participant's experiences, which were viewed from a distance. In this third-person perspective, three researchers looked at the visualized 
benefits of adaptive learning from an egocentric (subjective) perspective and tried to evaluate the usefulness of the idea of adaptive learning prototyping in higher education to build more sustainable personal knowledge (Buchenau, 2000).

Within the framework of the study, adaptive learning prototyping is seen as fishing skills of the individual in the 1PP action. To catch the fish, the individual can change the hook, the length of the fishing line, as well as use various types of squid. In order to make the catch successful and worthwhile in one's own understanding through sensitivity and sensibility relationship, the individual uses the personal experience, dynamically adapts to the environment, and, as a result, enriches, supplements and broadens the personal experience through the interaction with the environment.

In the case study, the researcher, on the basis of her personal exerience and value system, caught the most relevant ideas on the sustainability phenomenon from the summaries of doctoral theses. The main ideas corresponding to her personal point of view were placed in the centre of the diagram, followed by additional thoughts, impulses gained from the action research performed, which were located in the periphery of the drawing. These ideas also brough benefit to the comprehensive understanding of the sustainability phenomenon by the researcher.

\section{Figure 1}

Recognition of Sustainability in the Framework of the Egocentric Reference System

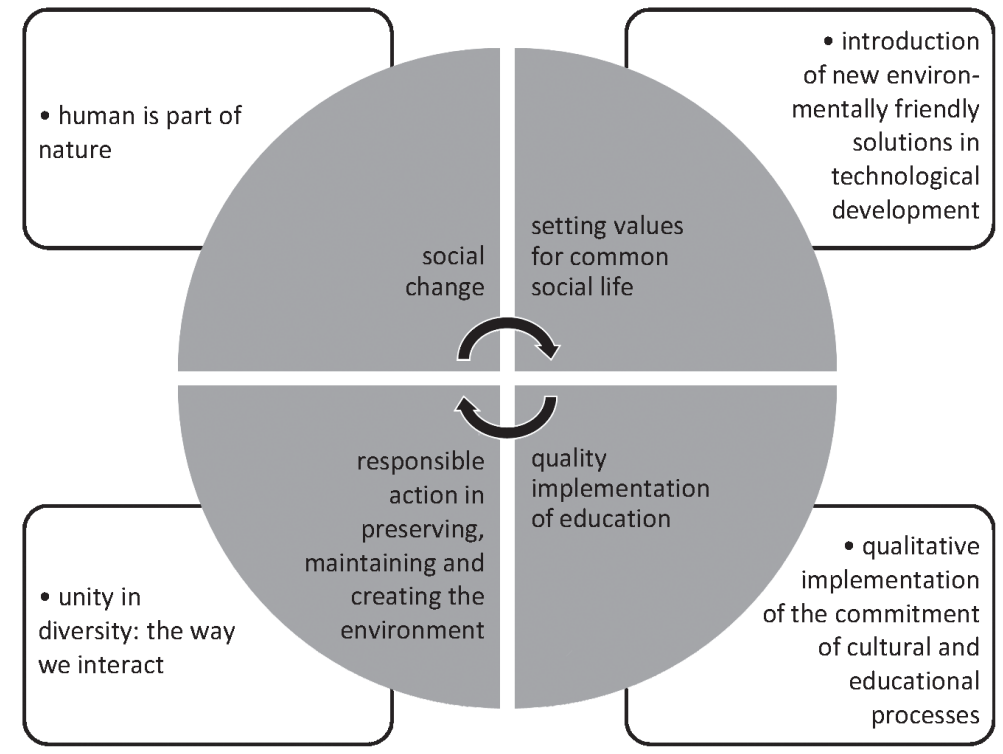

The structure of subjective experience from the researcher's point of view is placed at the center of the egocentric reference system experiences that are based on social change with setting values for common social activity, implementation of quality education and responsible action in preserving, maintaining and creating the environment. As seen in Figure 1, the periphery is filled with the notion that human is a part of nature, and there is a need for a high-quality connection and interaction of cultural and educational processes uniting diversity. 
The researcher identified the interrelation with her own experience, and demonstrated the fundamental basis for social change and typical current picture in education, common clichés, ideas currently characteristic of education policy that do not have a pronounced personal context; the relations between human and nature are conceptually hidden, because the part can be separated in different ways. The most common, familiar features were placed in the centre by the researcher, leaving the less significant items from the researcher's point of view aside.

\section{Adaptive Learning from Summaries of Doctoral Theses}

Adaptive learning, which had to be implemented remotely in a pandemic environment, was described in the previous section. Summaries of doctoral theses were used to expand the personally significant experiences. Evaluating the outcomes of this learning, we found out that the process of learning from the above-mentioned summaries was essentially a single approach (holism seeking sustainability) and the various studies revealed nuances that were natural in terms of methods, content, specific problems or ideas. In general, we associated this adaptive learning with the idea of prototyping. It appeared to be a natural idea for adaptive learning.

\section{Figure 2}

Extended Personal Knowledge on Sustainability Phenomenon Influenced by the Research of Grabouska (2006)

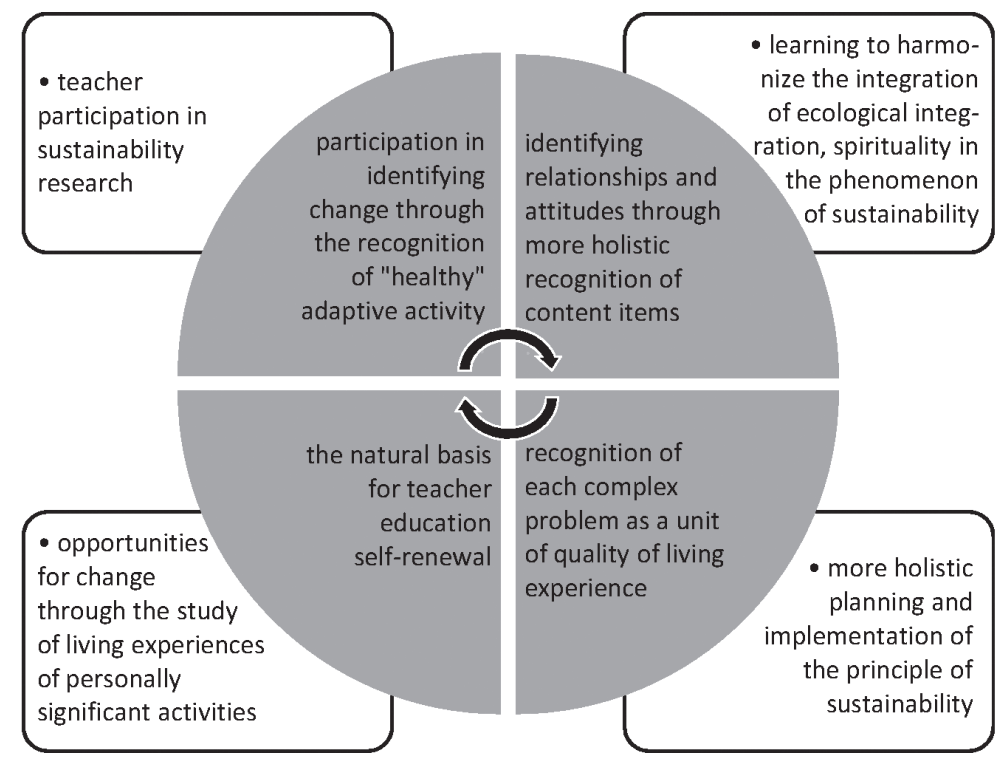

In this case, we can observe rapid opening of the "self" to participation, identification of signs of sustainability and attitudes through a more holistic unit of content and recognition of the quality of living experience, intended as a natural basis for educational self-renewal for teachers. 
The main finding arising from Figure 2 is that in the experience it is possible to actualize teachers' participation in sustainability research, learn to harmonize the content of ecology, integration, spirituality in the phenomenon of sustainability, as well as implement the principle of sustainability more holistically by introducing change through personal life experience research.

\section{Figure 3}

Extended Personal Knowledge on Sustainability Phenomenon Influenced by the Research of Gedžūne I. (2014)

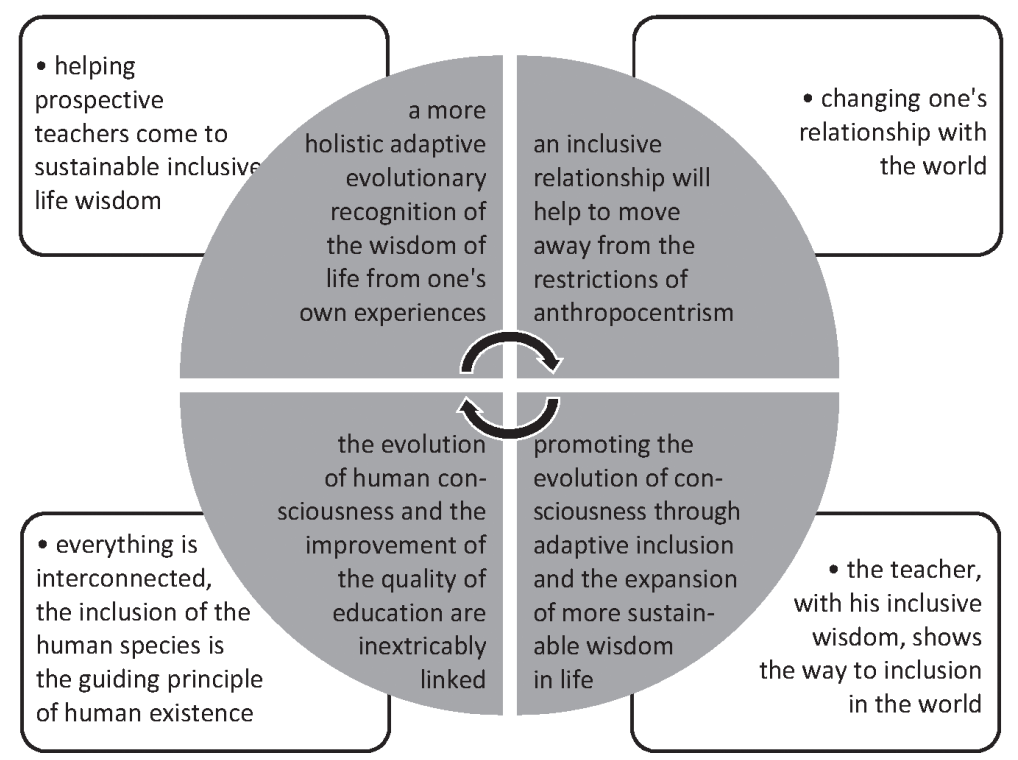

The researcher in the 1PP study identified a more holistic adaptive evolutionary recognition of the wisdom of life in one's experience, the impact of inclusive relationships on the reduction of anthropocentrism, adaptive inclusion, where the evolution of consciousness and the quality of education were inextricably linked (see Figure 3).

From the researcher's 1PP study, we can find the following valuable insights: it is of high importance to help prospective teachers come to the wisdom of sustainable, inclusive living, as well as change their relationship with the world, in which the teachers with their inclusive wisdom show the way to integrate in the world where everything is interrelated and the inclusion of the human species is the guiding principle of human existence.

The expansion of experience in this study is influenced by the recognition that a human being is a seeker of their own path through action and interaction; just writing and analyzing the issue of values is not enough; this is indicated by international documents and studies; the materialistic attitude of people and the use of resources are dominant.

People are looking for a path to sustainability, different levels of values form the basis of people's lives and social culture, at the beginning of the $21^{\text {st }}$ century the world is unsustainable and the lack of sustainability is caused by attitude (see Figure 4). 


\section{Figure 4}

Extended Personal Knowledge on Sustainability Phenomenon Influenced by the Research of Świtata (2015)

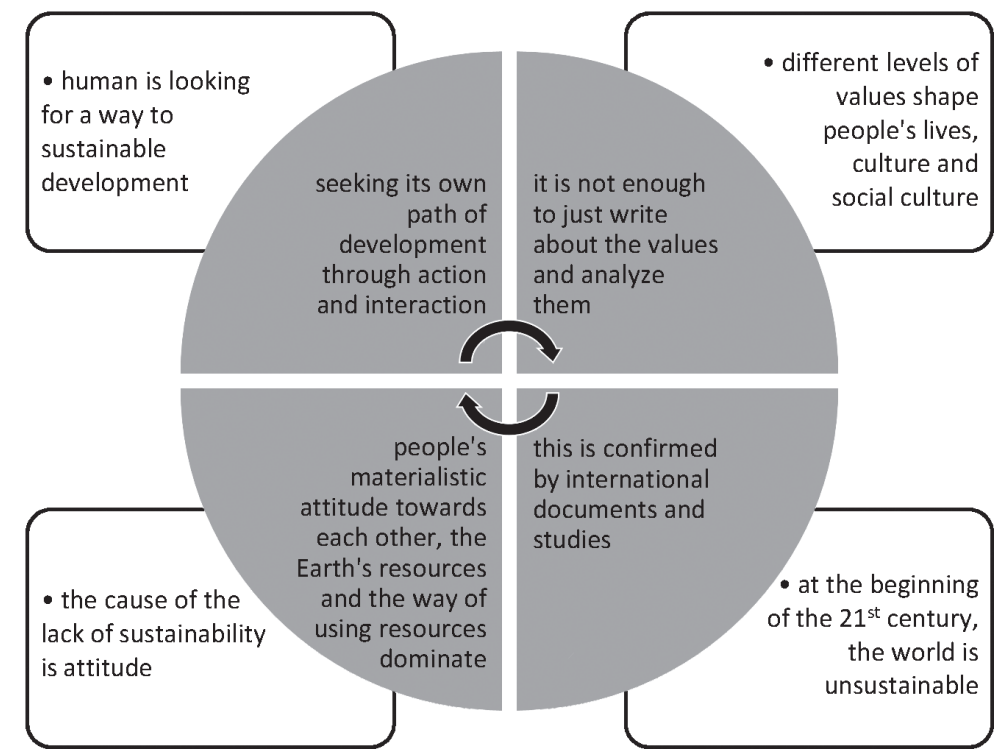

\section{Figure 5}

Extended Personal Knowledge on Sustainability Phenomenon Influenced by the Research of Gedžune G. (2014)

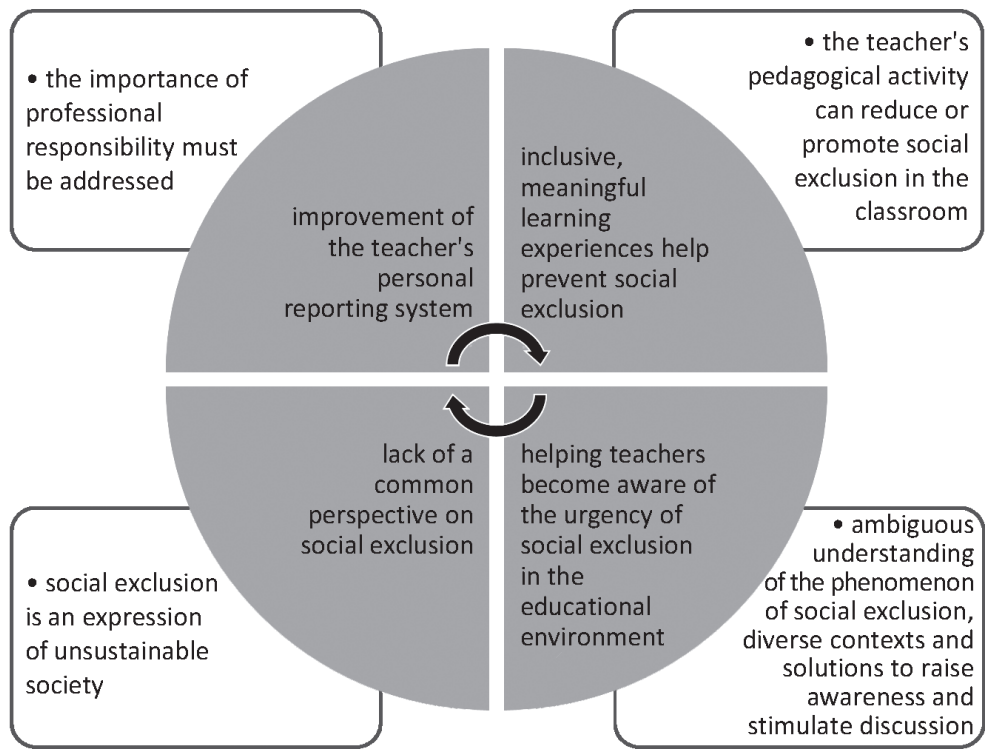


It is important to improve the teacher's personal reporting system, in which meaningful learning experience helps prevent social exclusion that in turn helps the teacher to be aware of social exclusion in the educational environment and the lack of a unified perspective on social exclusion.

From the researcher's 1PP study, we identify that sustainability also requires professional responsibility, as the professional activity of a teacher can reduce or promote social exclusion in the classroom, which is perceived differently as a phenomenon but which is an expression of an unsustainable society.

\section{Concluding Remarks}

Decision making, as associated with Aristotle, implied that a decision should be good and serve the majority; however, in the Anthropocene age the situation changed. The article has attempted to identify the most visible reasons why the following situation has arisen and what pedagogy can initiate in this regard.

The "tangle" of the problems discussed, in our opinion, is the result of Anthropocene, which also acts as a limiting force for sustainable development with a complex root based on unsustainability. The issues of this "tangle of Anthropocene problems" must therefore be seen in the light of the reasons for its unsustainability.

One issue of "the tangle of these problems" is the impact on people. The other easily identifiable issue of the Anthropocene problem tangle is the impact on the unsustainability of education. The third easily identifiable problem of the Anthropocene tangle is its easy transfer through the communication and technology environment and its use. In the three cases of the Anthropocene solutions, humanity has long recognized ecocentrism and deep ecology, the renewal of culture through the experience of individuals and the formation of meaning and self-development generated on its basis, which is a condition for the search for the highest goal of the human species. Humanity has sought to explain the world more widely, but in the Anthropocene conditions these efforts are undermined by the influence of its limiting forces.

We have been looking for an answer about the use of the 1PP study opportunities. However, it is not yet clear how we can influence the 1PP, as it can be hidden or falsified by the individual. It should be noted that sensitivity and sensibility are required for adaptive development and learning.

The research has revealed that personally important items can influence the depth of personal knowledge. And this can be achieved through the unique adaptive learning node, which is complex and in which the individual can look for meanings and values important personally to them.

The case study performed on the basis of summaries of doctoral theses has demonstrated that the personal experience connects and expands the personal framework for understanding of sustainability. This can be called prototyping, which can be used for adaptive learning purpose. This way, pedagogy can gain new experiences for a more holistic understanding of personal experience and enhance the use of the unique abilities of adaptive learning to prototype more personal knowledge in order to build more sustainable higher education. 


\section{References}

Aristotelis (1985). Nikomaha Ētika [Nicomachean Ethics]. Rìga: Zvaigzne. Blackie, J. S. (1883). The wisdom of Goethe. Charles Scribner's Sons.

Brigman, M., \& Lewis, J. (Eds.). (2017). The anthropology of sustainability: Beyond development and progress. Palgrave Macmillan.

Buchenau, M., \& Fulton Suri, J. (2000). Experience prototyping. Proceedings of the $3^{\text {rd }}$ conference on Designing interactive systems: Processes, practices, methods, and techniques, August, 2000, 424-433. https://doi.org/10.1145/347642.347802

Ferrare, P. (2019). Sustainable international relations. Pope Francis' Encicyclical Laudato Si' and the planetary implications of "Integral Ecology". Religions, 10, 466.

Gedžūne, I. (2014). Orientation of pre-service teachers' frames of reference towards inclusion in nature [unpublished doctoral dissertation]. Daugavpils University.

Gedžūne, G. (2014). Pre-service teachers’ frames of reference for addressing children's social exclusion in the classroom [unpublished doctoral dissertation]. Daugavpils University.

Grabovska, R. (2006). Implementation of the principle of sustainability in teacher education [Unpublished doctoral dissertation]. Daugavpils University.

Graziano, M. S. A. (2019). Rethinking consciousness: A scientific theory of subjective experience. W. W. Norton \& Company.

Hampton, J. A. (1991). The combination of prototype concepts. In P. J. Schwanenflugel (Ed.), The psychology of word meanings (pp. 91-116). Lawrence Erlbaum Associates, Inc.

Honkela, T., Hynnä, K. I., Lagus, K., \& Särelä, J. (Eds.). (2005). Adaptive and statistical approaches in conceptual modeling. Helsinki University of Technology.

McKenna, B. (2012). Greening anthropology. In Greening the academy. Ecopedagogy through the liberal arts (pp. 141-160). Brill.

Metzinger, T. (1995). Conscious experience. Imprint academic.

Morton, P. A., \& Mylopoulos, M. (Eds.). (2020). Philosophy of mind: Historical and contemporary perspectives. Broadview Press.

Perry, E. K., Collerton, D., LeBeau, F., \& Ashton, H. (Eds.). (2010). New horizons in the neuroscience of consciousness. John Benjamins Publishing Company.

Pigliucci, M., \& Kaplan, J. (2006). Making sense of evolution: Making conceptual foundations of evolutionary biology. University of Chicago Press.

Polanyi, M., \& Prosch, H. (1975). Meaning. The University of Chicago Press.

Revonsuo, A. (2009). Inner presence: Consciousness as a biological phenomenon. The MIT Press.

Rosenberg, G. (2004). A place for consciousness: Probing the deep structure of the natural world. Oxford University Press.

Salite, I. (1993). The raising of students' professional readiness for the ecological education mission at school, Doctoral thesis in Pedagogy, Latvian University, Riga.

Salite, I., Fjodorova, I., \& Ivanova, O. (2020). Editorial: Does the JTES help us create deeper personal meanings for sustainable education? Journal of Teacher Education for Sustainability, 22(2), 1-6. https://doi.org/10.2478/jtes-2020-0012

Salìte, I., Gedžūne, G., \& Gedžūne, I. (2009). Educational action research for sustainability: Seeking wisdom of insight in teacher education. Journal of Teacher Education for Sustainability, 11(2), 14-30. 
Snider, P. (2017). The natural problem of consciousness. De Gruyter.

Stratford, R. (2016, June 11). Towards a postfoundational ecological approach in higher education [Paper presentation]. The Sustainability and Education Policy Network (SEPN) Conference: University of Saskatchewan, Canada.

Stratford, R. (2019). Higher education and the Anthropocene - Towards an ecological approach to higher education policy in New Zealand. doi: 10.13140/RG.2.2. 28144.71685 .

Šmajs, J. (2008). Evolutionary ontology. Reclaiming the value of nature by transforming culture. Rodopi.

Świtała, E. (2015). Teachers' values related to sustainable development in Polish and Latvian secondary schools [Unpublished doctoral dissertation]. Daugavpils University.

Thompson, E. (2003). The problem of consciousness: New essays in phenomenological philosophy of mind. University of Calgary Press.

Wong, P. T. P. (Ed.). (2012). The human quest for meaning: Theories, research, and applications. Routledge.

Wright, P., \& McCarthy, J. (2010). Experience-centered design: Designers, users, and communities in dialogue. Morgan and Claypool Publishers.

Zanker, J. (2010). Sensation, perception and action: An evolutionary perspective. Palgrave Macmillan.

Zappalà, G. (2009). Spirituality is the new black ... and it has social impact! Part 1 Definitions and concepts. Working paper. The Center for Social Impact, 1-19.

Correspondence concerning this article should be addressed to Ilga Salite, Professor, Daugavpils University UNESCO Chair, Parades Street 1, 220, Daugavpils, LV-5401, Latvia. Email: ilga.salite@du.lv 